\title{
Article
}

\section{Railway Wireless Communications Channel Characterization}

\author{
Nerea Fernández-Berrueta ${ }^{1,2, *(\mathbb{D})}$, Jon Goya ${ }^{1,2}$, Jaione Arrizabalaga ${ }^{1,2}$, Iker Moya ${ }^{1,2}$ and Jaizki Mendizabal ${ }^{1,2}(\mathbb{D}$ \\ 1 Ceit-Basque Research and Technology Alliance (BRTA), Manuel Lardizabal 15, \\ 20018 Donostia/San Sebastián, Spain; jgoya@ceit.es (J.G.); jarrizabalaga@ceit.es (J.A.); imoya@ceit.es (I.M.); \\ jmendizabal@ceit.es (J.M.) \\ 2 Universidad de Navarra, Tecnun, Manuel Lardizabal 13, 20018 Donostia/San Sebastián, Spain \\ * Correspondence: nfernandez@ceit.es
}

Citation: Fernández-Berrueta, N.; Goya, J.; Arrizabalaga, J.; Moya, I.; Mendizabal, J. Railway Wireless Communications Channel

Characterization. Appl. Sci. 2022, 12, 345. https://doi.org/10.3390/ app12010345

Academic Editors: Nicola Bosso and Juan-Carlos Cano

Received: 20 September 2021

Accepted: 19 December 2021

Published: 30 December 2021

Publisher's Note: MDPI stays neutral with regard to jurisdictional claims in published maps and institutional affiliations.

Copyright: (C) 2021 by the authors. Licensee MDPI, Basel, Switzerland. This article is an open access article distributed under the terms and conditions of the Creative Commons Attribution (CC BY) license (https:// creativecommons.org/licenses/by/ $4.0 /)$.

\begin{abstract}
Railway applications are in continuous evolution with the aim of offering a more efficient, sustainable, and safer transportation system for the users. Generally, these applications are constantly exchanging information between the systems onboard the train and the trackside through a wireless communication. Nowadays, Global System for Mobile communications-Railway (GSM-R) is the technology used by European Train Control System (ETCS), but it is becoming obsolete. Therefore, alternatives for this technology have to be found for the different railway applications. Its natural evolution is to move forward with the latest technology deployed: Long-Term Evolution (LTE), which the Public Land Mobile Networks (PLMN) have already deployed. Therefore, testing the performance of this communication technology in the railway environment could be useful to assess its suitability and reduce the cost of railway network dedicated deployment. In order to do that, a methodology to characterize the communication environment is proposed. The main goal is to measure geolocated impairments of any communication channel in a railway environment being able to determine its behavior of the different communication technologies and find out possible coverage issues. Moreover, it could help in the selection of suitable communication technology for railway. This paper presents a brief description of the communication for railways and its QoS parameters for performance measuring. Afterward, the testing methodology is described, and then, the communication channel measurement campaign on a real track in Spain where the railway environment is variable is presented (tunnels, rural/urban area ... ). Finally, the measurements and results on this real track in Spain are shown. The results provide suitability of the $4 \mathrm{G}$ technologies based on the delay requirements for the implementation of ETCS over it.
\end{abstract}

Keywords: communication channel; IP impairments; measurements; railway environment; railways

\section{Railway Communications and Evolution}

Nowadays, more and more people use the train as an option for transport such as subways, tramways, or high-speed trains. Because of the importance of the increasing amount of people that choose the train, the transportation system has to obtain certain safety guarantees in order not to put the passengers' lives at risk, as well as offering a number of services to the passengers. To reach this main goal, railway applications are in continuous evolution. These applications are more sophisticated and restrictive in terms of Quality of Service (QoS), such as real-time applications that need low delay and jitter. In addition, not every application needs the same requirements: the safety-related applications are more restrictive than the non-safety or passenger-comfort-related applications. A large amount of these applications uses the communication channel between the onboard and trackside to exchange information about the train. Moreover, depending on the application, the technology of the communication channel is not the same. For example, CommunicationsBased Train Control (CBTC), a signaling system for subway trains, based on IEEE 802.11 [1], makes use of different communication technologies, in constant evolution depending on the manufacturer. By contrast, the selected signaling system for the mainline and high-speed 
rail in Europe, the European Rail Traffic Management System (ERTMS), uses Global System for Mobile communications-Railway (GSM-R). Some of the limitations of GSM-R are [2]:

1. Low link rate: its maximum transmission rate per connection (9.6 kbps) is sufficient only for applications with low demands (ETCS is around $4 \mathrm{kbps}$ ). Moreover, its delay, which is the range of $400 \mathrm{~ms}$, is too high for critical video applications whose acceptable delay is $100 \mathrm{~ms}$.

2. Interferences with the GSM-R due to different sources:

a. Electromagnetic (EM) transient interferences due to the sliding contact between the pantograph and the catenary [3].

b. Public GSM communications employ the channels adjacent to the GSM-R frequency [4].

3. Small system capacity: GSM-R system with the available $4 \mathrm{MHz}$ bandwidth can only support 19 channels of $0.2 \mathrm{MHz}$ bandwidth per channel, which cannot accommodate the rapidly developing railway communications [5].

Taking into account these limitations it can be stated that GSM-R technology is becoming obsolete, with an end of support planned by 2030 onwards. Since it does not accomplish the requirements for the current applications, some other technologies shall be used as its substitute. One of the candidate technologies is Long-Term Evolution (LTE), which is supposed to be the natural evolution of GSM-R as it is the current evolution in the public operators [2,6]. However, there are also other suitable technologies either deployed or in progress, such as satellite communications or $5 \mathrm{G}$, respectively.

Nevertheless, the deployment and maintenance of a railway-dedicated network are really expensive; therefore, the decision of deploying a new network has to fulfill some guarantees. As the Public Land Mobile Network (PLMN) operators have already deployed a number of technologies, they could shed light on railways in their environment to know how the communication channel is. Particularly, the railway environment could affect the communication channel because of EM disturbances or the environment where the train is passing through (tunnels, rural areas, urban areas, etc.). Then, a system capable of measuring the current technologies already deployed arises from the need of knowing how the communication channel is and how it will behave in the railway environment, and with these results, finally, one can decide if it is worth deploying a new dedicated network or not. As a solution to this issue, a novel tool called the Channel Characterization Tool (CCT) is described in this paper to characterize the performance of a wireless communication link to check specifications and bring the real environment to the laboratory.

The main contributions of this paper are:

- Description of the QoS parameters for communication technology performance measuring and the different railway environments that exist.

- Comparison of different tools in the market for measuring the communication channel.

- A methodology for testing the communication technologies.

- Innovative tool capable of measuring different IP impairments, geolocating them into the railway network.

- Performance results of the real environment field-test to validate the usage of the deployment.

First, the QoS parameters for communication technology performance measuring are explained (Section 2). Then, some of the tools to measure the communication channel available in the current market are exposed (Section 3). Moreover, the testing methodology for communication channel measurement and the innovative tool called CCT are explained (functions, architecture) (Section 4). Furthermore, some typical characterization scenarios of railways are included (Section 5). Later, a deployment example is described and shows the results obtained from the test campaign (Section 6). Finally, conclusions are drawn (Section 7). 


\section{QoS Parameters for Communication Technology Performance Measuring}

The communication channel is the part of a system that allows sending information from the transmitter to the receiver. It can be characterized regarding different concepts such as radiofrequency or IP level. Focusing on IP level, the main IP QoS parameters that define a communication channel are the following ones:

- $\quad$ RTT: duration required for a network to send a request from a starting point to a destination and back again to the starting point.

$$
R T T=t_{1}+t_{2}
$$

where $t_{1}$ is the time required to a packet from the source to the destination, and $t_{2}$ is the time required to a packet from the destination to the source.

- Jitter: measurement of the latency variation above and below the mean latency value. The maximum jitter is defined as the maximum latency variation above and below the mean latency value [7].

- Bandwidth: in terms of data network, bandwidth quantifies the data rate at which a network link or a network path can transfer; the amount of data a link or network path can deliver per unit of time [8].

$$
B W(\text { bps })=\frac{\sum \text { bits }}{\text { second }}
$$

- Packet loss: the failure of a packet to traverse the network to its destination [9,10]; it is measured as the percentage of lost packets over the sent ones. It occurs when one or more packets transmitted over an IP network fail to arrive at their destination. Packet loss is typically caused by what is generally referred to as network congestion, but also because of distance or poor line quality. Excessive packet loss is perceived as disconnections (broken or missing communication).

$$
\text { Packet loss }(\%)=\frac{1-\sum \frac{\text { received packets }}{\text { sent packets }}}{\text { second }} * 100
$$

- Throughput [11]: the number of payload bits successfully received per second for a reference measurement channel in a specified reference condition.

$$
\text { Thoughput }(\text { bps })=\frac{\text { payload bits }}{\text { second }}
$$

- Disconnections: a time interval where there is no connection. One of the reasons could be the lack of coverage.

Moreover, regarding radiofrequency scope for wireless communication, other parameters exist that allow to find out the behavior of the communication channel, such as:

- Received Signal Strength Indicator (RSSI): defined as the power of a certain signal of interest divided by the sum of the interference power (from all the other interfering signals) and the power of some background noise.

Furthermore, other parameters are specific for technologies, e.g., for LTE, such as:

- Signal-to-interference-plus-noise-ratio (SINR): defined as the power of a certain signal of interest divided by the sum of the interference power (from all the other interfering signals) and the power of some background noise.

$$
\mathrm{SINR}=\frac{S}{I+N}
$$

where $S$ is the desired Signal $(\mathrm{W}), I$ is the interference $(\mathrm{W})$, and $N$ is the noise $(\mathrm{W})$. 
- $\quad$ Reference Signal Received Power (RSRP): defined as the linear average over the power contributions (in $(\mathrm{W})$ ) of the resource elements that carry cell-specific reference signals within the considered measurement frequency bandwidth [12].

- $\quad$ Reference Signal Received Quality (RSRQ): defined as the ratio $N \times$ RSRP/(E-UTRA carrier RSSI), where $N$ is the number of RBs of the E-UTRA carrier RSSI measurement bandwidth [12].

$$
\mathrm{RSRQ}=\frac{N \times \mathrm{RSRP}}{\text { LTE carrier RSSI }}
$$

where $N$ is the number of Resource Blocks of the LTE carrier of RSSI measurement bandwidth.

\section{Characterization Tools}

The mentioned QoS parameters shall be measured in the proposed environment in order to characterize the communication channel. In fact, the mapping of the different QoS parameters and the corresponding impairments with the location is relevant information in order to know how the communication channel is and where the environment could affect it. Thus, the reason for possible degradation of the communication channel or even the lack of coverage can be known thanks to the mapping of location-impairment. For that, three tools available in the market are compared and shown in Table 1: R\&S products for drive test software, Pilot Pioneer from DingLicom, and RantCell.

Table 1. Tool comparison.

\begin{tabular}{|c|c|c|c|}
\hline & R\&S [13] & DingLicom [14] & RantCell [15] \\
\hline Networks to be measured & $\begin{array}{c}\text { 5G NR } \\
\text { Nb-IoT/Cat-NB1 } \\
\text { GSM/EDGE } \\
\text { WCDMA/HSPA+ } \\
\text { CDMA2000 } \\
\text { WiMAX } \\
\text { LTE } \\
\text { LTE-M } \\
\text { TETRA } \\
\text { WLAN }\end{array}$ & $\begin{array}{c}5 \mathrm{G} \\
4.5 \mathrm{G} \\
4 \mathrm{G} \\
3 \mathrm{G} \\
2 \mathrm{G} \\
\text { LTE-IoT }\end{array}$ & $\begin{array}{l}5 G \\
4 G \\
3 G \\
2 G\end{array}$ \\
\hline Environment & Any environment & Any environment & Any environment \\
\hline Measured parameters & $\begin{array}{c}\text { RF (SINR, RSRQ, RSRP) } \\
\text { QoS testing capabilities: } \\
\text { coverage measurements, } \\
\text { interference identification, } \\
\text { performance measurements, } \\
\text { quality analysis in mobile networks }\end{array}$ & $\begin{array}{c}\text { RF (SINR, RSRQ, } \\
\text { RSRP) } \\
\text { Network Coverage, } \\
\text { Qualified Coverage, } \\
\text { Difference Value, } \\
\text { Effective Cells, Cell } \\
\text { Handover, } \\
\text { Bandwidth, } \\
\text { Location }\end{array}$ & $\begin{array}{l}\text { RF (RSSI, RSRP, RSRQ, } \\
\text { among others), } \\
\text { Latency, } \\
\text { Bandwidth (separated } \\
\text { points), } \\
\text { Location }\end{array}$ \\
\hline $\begin{array}{l}\text { Testing and } \\
\text { post-processing/visualizing } \\
\text { from the user point of view }\end{array}$ & $\begin{array}{c}\text { ROMES4 } \\
\text { R\&S TSMx scanners } \\
\text { R\&S }{ }^{\circledR \circledR} \text { ROMES4NPA } \\
\text { Test mobile devices (up to } 8 \text { at the same time) } \\
\text { Additional devices/drivers depending on } \\
\text { the use case to be tested }\end{array}$ & $\begin{array}{l}\text { Testing: Pilot Pioneer } \\
\text { Post-processing and } \\
\text { visualizing: Pilot } \\
\text { Pioneer Expert }\end{array}$ & $\begin{array}{c}\text { Smartphone needed (it } \\
\text { has a list of support } \\
\text { devices) }\end{array}$ \\
\hline User-customizable & - & - & - \\
\hline
\end{tabular}

As Table 1 shows, these tools can measure different technologies and parameters in any environment. Nevertheless, they are not a user-customizable tool, technologies are fixed, and there is no chance to integrate another current of future technologies in the system. Additionally, the system hardware set-up-more particularly, the antennas-cannot be 
changed, reducing the capabilities of measuring the impact of the different antennas during the measurements and avoiding the use of higher gain antennas.

Furthermore, some of these tools need the purchase of more than one product of the same company instead of having an all-in-one device or one service for the whole test performance.

Moreover, these tools can be employed in any environment as they are not railwayspecific, so they would not consider the railway specific track with the corresponding stations.

Regarding how to obtain and visualize the results, the DingLicom tool requires another product for the post-processing and visualizing task. Moreover, if the goal is to measure different environments at the same moment, $R \& S$ needs multiple products to reach the same goal present in the $R \& S$ products.

As well, some of these tools can measure specific impairments such as maximum data rate with a location, but not in a continuous mode; they are rather individual tests in separated moments, and therefore, separated locations. The aim of having a continuous geolocated impairment is not covered by any of these tools.

In conclusion, these tools do not completely fulfill the purpose of the railway migration task. Because of these reasons, an innovative tool called Channel Characterization Tool (CCT) is proposed.

\section{Communication Channel Measurement Methodology and CCT Description}

CCT is a technology-independent tool, which allows the measurement of different communication channels in terms of IP QoS parameters along a given track with a specific location. Intending to fulfill this main requirement, the methodology proposed is the following one:

1. Installation of an on-board device in the train.

2. Configuration of the different tests to be performed in the specific track.

3. Test measurements of the different IP impairments with the need of being geolocated to obtain information on how the communication channel behaves in each location of the tack.

4. Processing of the test results.

5. Visualization in a user-friendly interface.

6. Analysis of the results of the test.

In order to reach the main goal already mentioned, the development of the CCT has been carried out. The main features of this tool are explained in Table 2.

Table 2. CCT features (innovations with respect to state of the art are marked with *).

\begin{tabular}{|c|c|}
\hline & CCT \\
\hline Networks to be measured & $\begin{array}{l}\text { Any network the user wants to monitor, the } \\
\text { CCT is plug and play * }\end{array}$ \\
\hline Environment & $\begin{array}{l}\text { Any environment, with railway-specific } \\
\text { module * }\end{array}$ \\
\hline Measured parameters & $\begin{array}{l}\text { RF and IP parameters: BW, throughput, RTT, } \\
\text { jitter, packet loss, RSSI, RSRP, RSRQ, SINR }\end{array}$ \\
\hline Usage & $\begin{array}{l}\text { Easy usage adapted also for } \\
\text { non-communication expert users * }\end{array}$ \\
\hline $\begin{array}{l}\text { Testing and post-processing/visualizing from } \\
\text { the user point of view }\end{array}$ & $\begin{array}{l}\text { On-board testing equipment: CCT agent } \\
\text { Visualizing: internet connection and web page } \\
\text { User-friendly interface }\end{array}$ \\
\hline User-customizable & $\begin{array}{c}\text { Any technology can be inserted * } \\
\text { Customizable connection for any technology } \\
\text { (Ethernet connection) }{ }^{*} \\
\text { SMA connections for desired antenna }\end{array}$ \\
\hline
\end{tabular}


In comparison with the other tools already mentioned, this novel tool allows testing the communication channel in continuous mode along a track and not point by point like static testing. Moreover, it is a save-spacing tool in addition to being a non-intrusive system; it can work in a train in operation, not degrading its normal functioning.

Hereunder, some of the benefits of CCT are detailed:

- $\quad$ CCT measures the impairments defined by the user in a continuous mode with its geolocation. This feature is the most important one since the railway environment changes while the train is going from the starting point to the endpoint of the journey, affecting the communication channel.

- $\quad$ CCT is a modular and plug-and-play tool; therefore, the CCT can be networkcustomized, allowing the possibility to include current technologies that are not integrated such as satellite communications or in development (e.g., 5G) or future ones such as 6G. Furthermore, other technologies which other purposes such as maintenance could be tested to know their limitations, such as LoRA which is being used in the railway domain [16].

- CCT contains information about the different network operators such as theoretical coverage (cell information), which could help in the analysis of the network performance.

- $\quad$ CCT is railway specialized; therefore, the mapping of QoS parameters is more accurate along the track than in general applications.

- This tool includes more IP impairments than the other tool in the current market: packet loss, jitter, throughput.

- CCT allows an easy usage for expert and non-expert users in the communication area.

- It allows for knowing the statistic results from every test and impairment, visualizing the summary of how the communication channel is based on the different tests performed in the same track.

- A user-friendly interface for configuring and visualizing the tests is included, being accessible from any device with an internet connection.

- The antennas to test the communication channel can be switched as the connection with the antenna consists of an SMA connector. Consequently, different antennas can be plugged into the system, changing, e.g., the gain of it (dB).

Thus, CCT is a specific railway tool which allows accurate geolocated communication channel measurements adaptable to any track or technology.

The CCT architecture consists of three different parts split into on-board and trackside, shown in Figure 1.

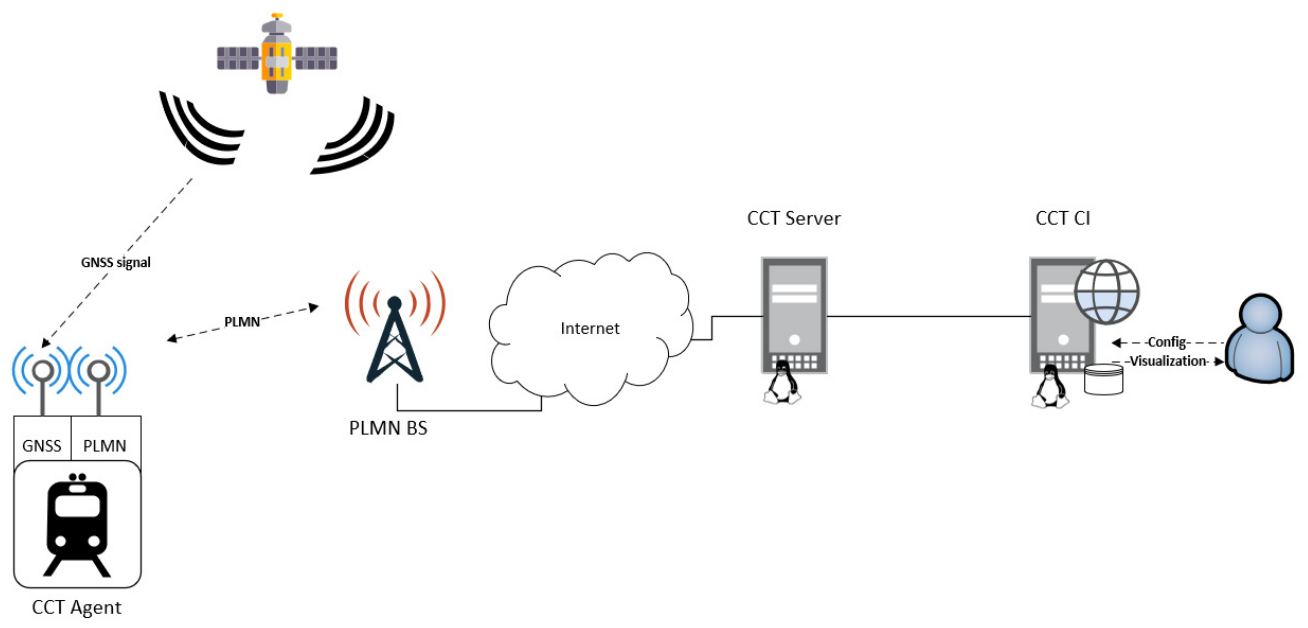

Figure 1. CCT architecture.

In the CCT, the user is responsible for taking the control of the whole system by CCT $\mathrm{CI}$, configuring the tests and visualizing their results. The CCT agent and server are the 
parts of the system that allow the testing of the already configured communication channel. Moreover, the CCT Agent is the part installed on the train, highlighting its easy installation and start-up and being space-saving due to the small size of the device.

The functions of each part of the system are explained below.

- CCT_Agent: the on-board part located on the train. It is the part responsible for obtaining the train position and monitoring the parameters of the communications configured from CCT_CI. It can operate from trains in operation, being a non-intrusive system, to dedicated vehicles. Moreover, it is a scalable system with regards to the number of trains on which it is installed. In addition, it adapts to wireless communications technologies to be monitored: 2G, 3G, and 4G (current ones); 5G (in development); Tetra; WIFI; SATCOM; ITS-G5; and so on.

Figure 2 shows the connections of the CCT agent. Concerning the technology to be monitored, SubMiniature version A (SMA) connection is available to plug the antenna for the desired technology to be measured (e.g., it could be a multiband antenna for $3 G$ and $4 \mathrm{G}$ ) and as well the Ethernet connection that allows the user to plug the tool into a specific network.

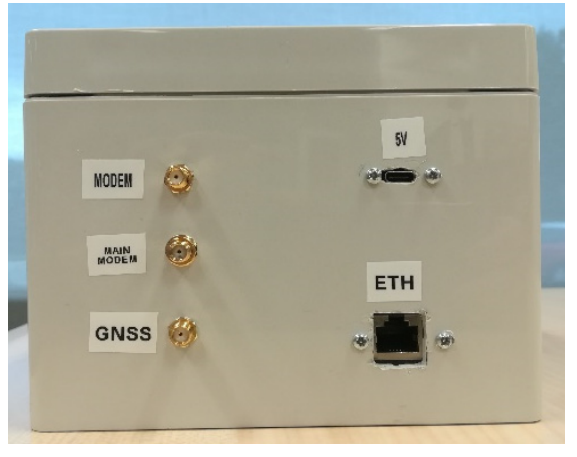

(a) CCT Agent

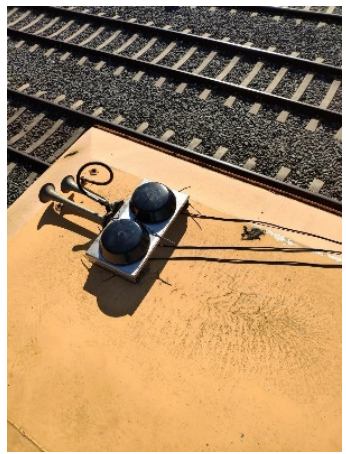

(b) GPS antenna on the roof of the train

Figure 2. On-board installation of the CCT Agent.

- CCT_Server: it manages the information of CCT_Agent and CCT_CI, acting as an interface between both systems. It is responsible for processing and analyzing the information from the test to obtain the desired parameters that the user configured. The location of this part of the CCT differs depending on the use; if the philosophy to follow is being tested in a specific area in a country, the server could be installed locally. However, the CCT can be performed in multiple tracks around the world with the need to connect to the same server; therefore, the cloud is the other option that can be chosen due to its elasticity property which allows the cloud platforms to efficiently add or remove the cloud infrastructures automatically according to the number of users for supporting the rapid fluctuation of loads to serve better [17]. For this specific case, the CCT_Server is located in the cloud.

- $\quad$ CCT_CI (Control Interface): it is the configuration part done by the final user regarding the test that is going to be executed. In addition, it owns a database where routes, trains, monitored parameters, etc., are included. These data will be needed for the understanding of the final results. Another task for this CCT part is the visualization of the test results, which are shown in different formats such as maps or graphics:

Configuration of each of the CCT_Agent(s) to activate/deactivate them to monitor the parameters defined in the time interval that the user wants.

Visualization: The user can visualize the results in different display modes:

Maps: the web interface shows in a map the different values along the track where the train was passing through during the test.

- Graphics/charts: the CI shows charts comparing time vs. value. 
KPI chart: the quality of service of each application to work properly in chart form. In this way, this chart can be compared with the other charts from the test, letting the user know which applications will work or not (comparison with one impairment) [18].

For example, some typical Key System Requirements from the main railway applications in terms of latency are listed in Table 3.

Table 3. KSR from some applications [18].

\begin{tabular}{cc}
\hline KSR & Max. Latency (s) \\
\hline ETCS & 3.5 \\
CBTC & 0.1 \\
Critical Voice & 0.15 \\
Critical data & 0.1 \\
Critical Video & 0.1 \\
\hline
\end{tabular}

In order to well understand how the system works, a sequence diagram of the communication between the parts of the systems is shown in Figure 3.

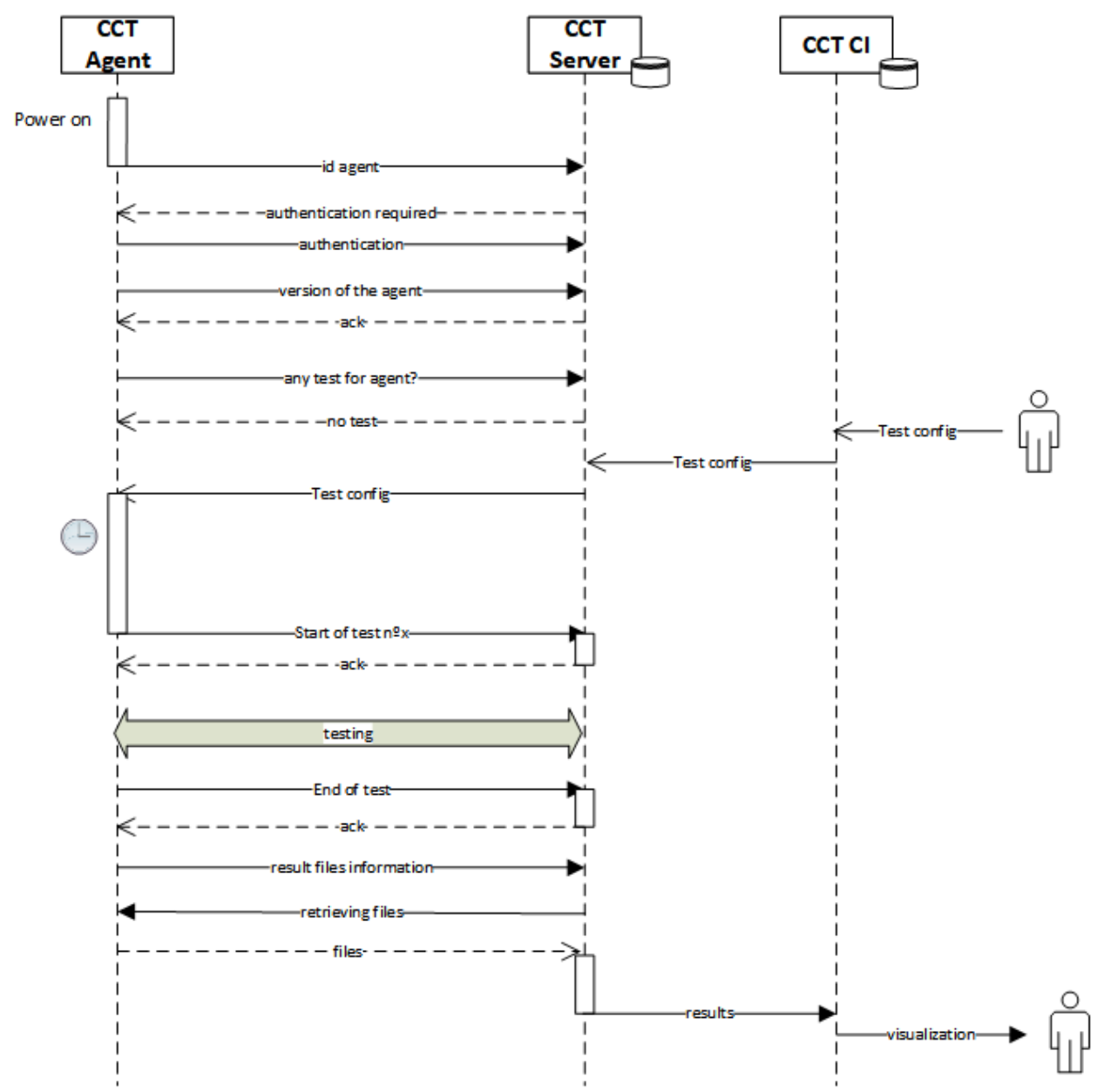

Figure 3. Sequence diagram between CCT Agent, CCT Server, and CCT CI.

The CCT Agent and CCT Server are in continuous communication in order to start and end the test at the hour already configured by the user, to perform the desired test, and to send the result files. However, the CCT Server communicates with the CI in the configuration and after processing the files, sending the results. 
These three parts of the system allow measuring the different communication channels and analyzing their results. In this case, the measurements will be carried out on a real track in Spain.

\section{Characterization Scenarios}

The desired signal sent through the communication channel could be affected by different situations given along a track. Some of these different situations are the following ones:

- Railway environment;

- PLMN;

- Handovers;

- EM disturbances.

The environment that the train is passing through is an important element to take into account. Because of the environment, the desired signal can be perturbed by different effects as Figure 4 shows, such as multipath or scatters.

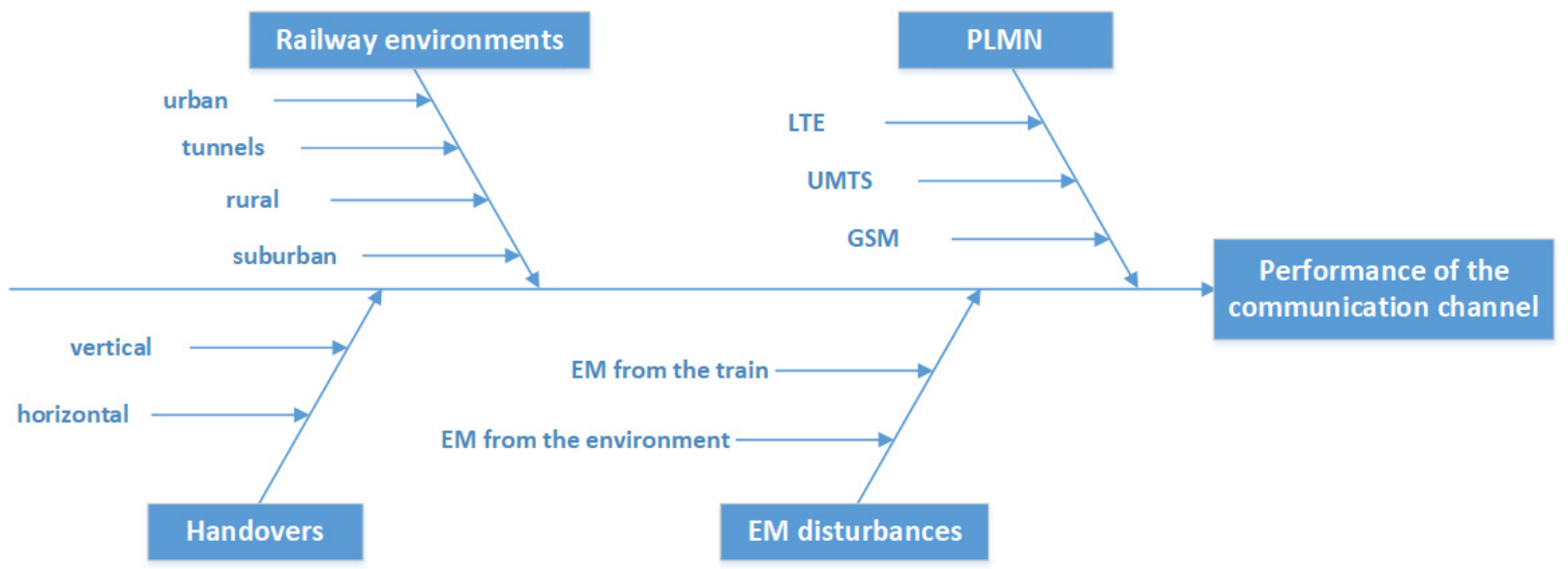

Figure 4. Fishbone of the effects that affect the performance of the communication channel.

Some possible scenarios that cause these effects are [19]:

- Open Space:

Urban area: normally many antennas are placed in this area because of the high amount of population, and consequently, more coverage. However, there are many obstacles which are characteristic of this area, the most often seen ones being buildings, barriers, ground, tracks, tractions, and trains [20].

Rural area: fewer obstacles than the urban area; therefore, better line-of-sight (LoS), but less coverage. The transmit antenna is much higher than the surroundings which are light forests adjacent to the track (crops less than $2 \mathrm{~m}$ ) and a few buildings with an average height of less than $10 \mathrm{~m}$. The link between the transmitter and receiver generally has a strong LoS component. However, after a certain distance, if there is no line-of-sight (NLoS), scatterers will be noticed at the receiver [21,22].

Suburban area: where the buildings are typically low residential or townhouses with one or few floors [22]. Occasional uncluttered areas make the environment rather open, and the vegetation is modest. In fact, for High-Speed Railway (HSR) lines, most vegetation and buildings are $300 \mathrm{~m}$ away from the rail tracks. Tunnels: normally no coverage inside the tunnel because there are no available antennas inside.

- Viaduct: one of the most common scenarios for railways, especially for HSR [22,23]. The viaduct raises the antennas and therefore creates a relatively "clear" LoS channel, 
thus reducing the number of the scatterers that can affect the fading and having as result an LoS propagation and few received reflection and scattering components.

- Cutting (also called U-shape cutting): a semi-enclosed construction usually with two slopes along the track, constituting a common scenario in HSR environments [23,24]. The propagation is significantly affected by the steep walls on both sides [25], generating typically scatters.

- Station: classified according to their size or architecture [25]: open station, semi-closed station, and closed station. The huge steel awnings covering the platform and track, steel frames beside and above the track, and metallic pylons at the front side and the broadside of the station can block parts of LoS between the onboard receiver and the transmitting antenna outside the station. Consequently, the propagation suffers extra loss when the train is inside or near the station [26].

The CCT uses, among other possibilities, the PLMN as they have already deployed some technologies such as LTE, which is a possibility for railways. In this way, the behavior of these technologies in railways can be researched. Therefore, there is another important factor to take into account. Because of using the PLMN, different impairments can affect the signal. On one hand, each operator can offer a specific coverage, which is different from other operators due to the location and the power of the managed antennas. On the other hand, the number of users wanting to connect to the network at the same time indirectly affects the communication channel. This could be the reason that the network redirects one user to a further antenna and not the one closest to the track, which causes a worse communication channel, even no coverage.

In addition, some problems related to the connection can appear due to handovers, mostly in HSR. Because of its high mobility, there are three main problems with handover: unstable signal quality, group handover, and high-frequency handover [27].

Moreover, EM disturbances are present in railway environments, not only in GSM-R as Section 1 introduced but also in other technologies such as LTE-R. One of these EM disturbances is the arcing between the pantograph and the catenary that produces a considerable EM interference in the LTE-R (in 400MHz frequency band), resulting in transmission errors as [28] states. As [29] states as well, the susceptibility of LTE communication when faced with EM interferences (catenary-pantograph sparks) present onboard trains is not constant and depends on the LTE communication channel bandwidth.

Because of the already mentioned different situations in railways, the communication performance is affected.

\section{Deployment Example}

The CCT can measure any communication channel in any railway track, helping to go forward in the decision of migrating from current technologies to new ones because of the current capabilities. Moreover, the tool can help a specific user develop a railway application on how this application behaves in a specific track due to the network deployed and the railway environment. As shown in Section 2, CCT measures different parameters, some of which, depending on the application to be used, will be more critical than others. For example, regarding the requirements of each application indicated in [18], ETCS is a critical application, for which the minimum requirements are a data rate of $4 \mathrm{kbps}$ and a latency of $3.5 \mathrm{~s}$ [18], but only a probability of data loss less than $10^{-4}$ [30]. However, a real-time critical video application needs a minimum data rate of $5 \mathrm{Mbps}$ as the amount of data for video streaming tends to be higher than ETCS, the constant delay should not exceed $0.1 \mathrm{~s}$, and the acceptable packet loss should be around 4\% [31].

As an example of the tests and results given by the CCT, a test campaign has been carried out in a specific track placed in Spain, more specifically, in the track Ciudad RealCerro Negro. This track has several railway environments causing different effects on the desired signal. During the test campaign, the different parameters that the CCT is capable of measuring have been tested: RTT, jitter, bandwidth, throughput, radio frequency values such as RSSI, and packet loss. However, the results shown are from one of the available 
parameters: RTT. The rest of the parameters have the same visualization options, but the RTT results are shown as an example. Additionally, the results correspond to a part of the whole track mentioned before, being this sub-track placed between Almonacid de Toledo and Mora and the wireless technology provided by the Vodafone network operator.

The environment that was identified for this specific track where the train is passing through is mainly a suburban area, as Figure 5 shows.

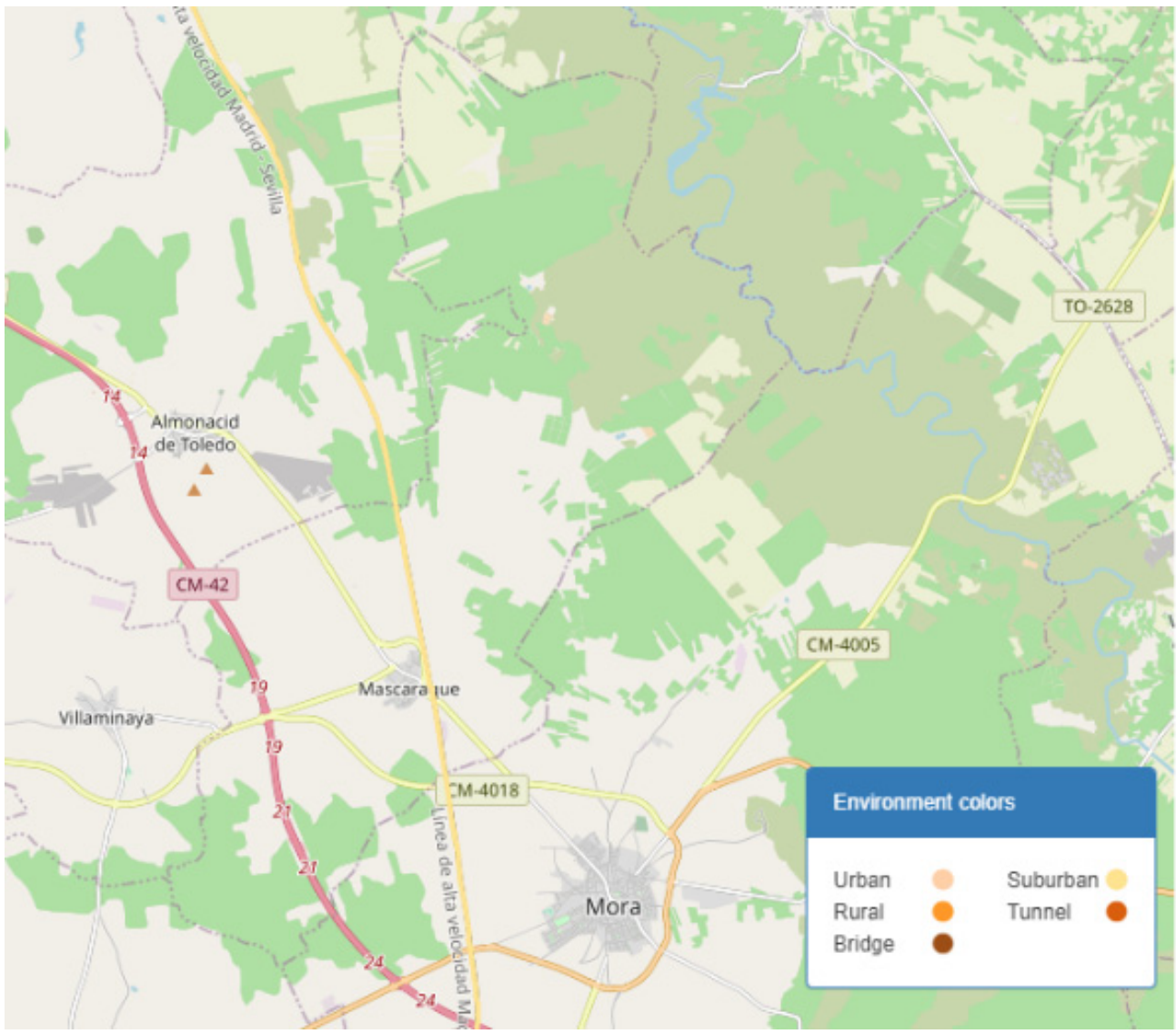

Figure 5. Measured track: Ciudad Real-Cerro Negro.

Some results obtained by the testing of the different parameters are shown in this section using the visualization part of the CCT, that is, CCT CI.

Apart from these interesting values obtained from the test campaign measurement, the powerful CCT system can provide more information that helps the user understand what happened during the test campaign, such as the cells from the network operator that the agent was attached to or the technology measured in each point.

The CCT allows the user to both configure the test and visualize the results. The visualization functionality permits the user to know the quality of the communication channel while on-site testing for each location in the track, as Figure 4 shows.

As Figure 6 shows, the RTT is, in general, around 100 ms. However, there are some points in the track that have different values, being greater than the average. This effect could be because of the environment affecting the communication channel or the proximity to the cell. 


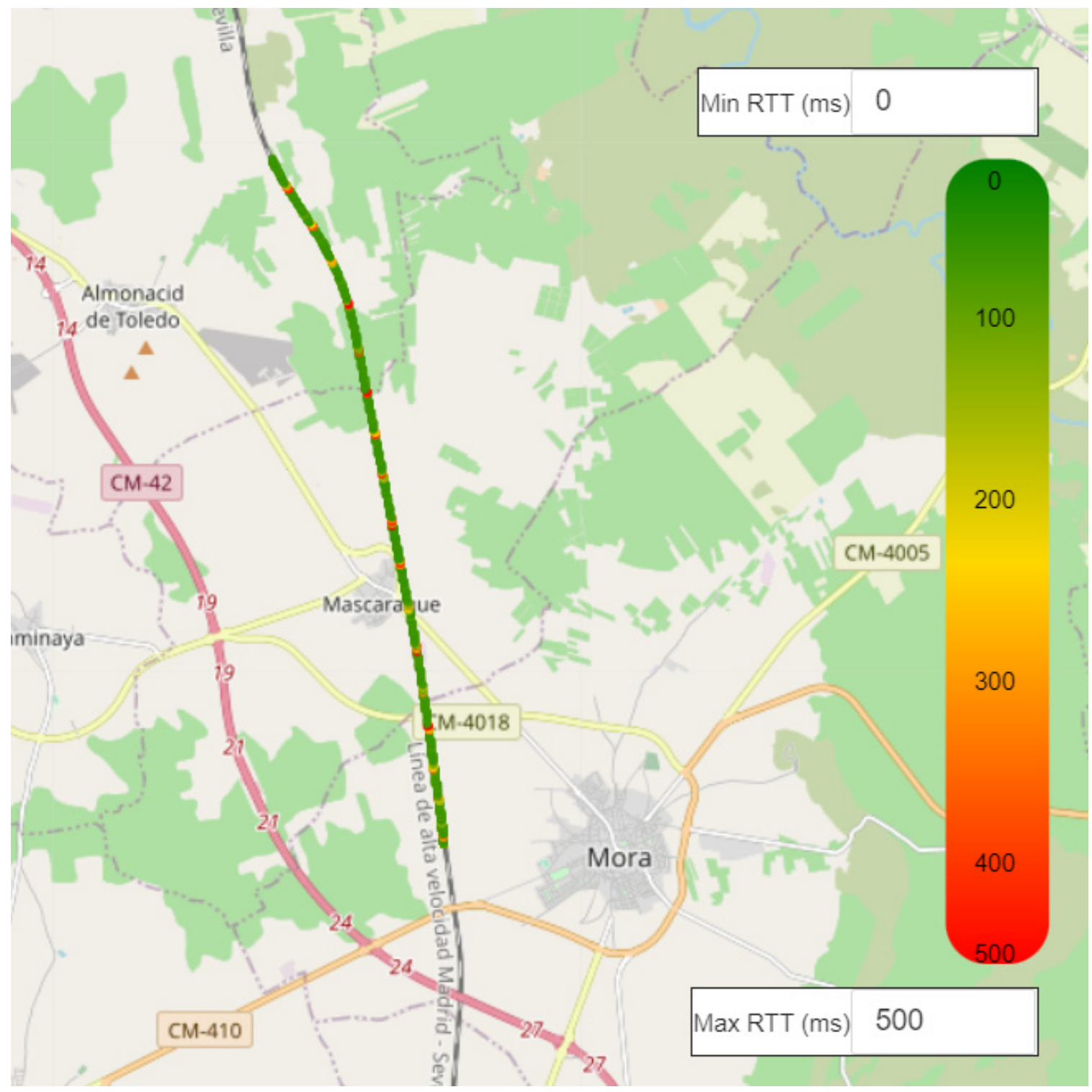

Figure 6. Round Trip Time test.

Furthermore, the tool can generate a coverage map to find out the lack of coverage or the available technologies in the specific track, as Figure 7 shows. In this specific case, the CCT had total LTE coverage in the track, resulting in no vertical handovers and no disconnections from the fact of switching the technology.

Moreover, this tool allows both comparing different tests between each other and comparing the same test with different conditions ( $\mathrm{min}$ and max values changed) at the same moment as Figure 8 shows or in different tests.

Furthermore, the CCT CI can show statistics results, that is, the results of a given test sent by the user or the results of the different tests performed in a given track as Figure 9 shows. The results of the different tests are customizable in terms of visualizing the average, the number of measurements, and the maximum or the minimum value (see Figure 9), and it can also decide the size of the area where the previous options have to be applied. 


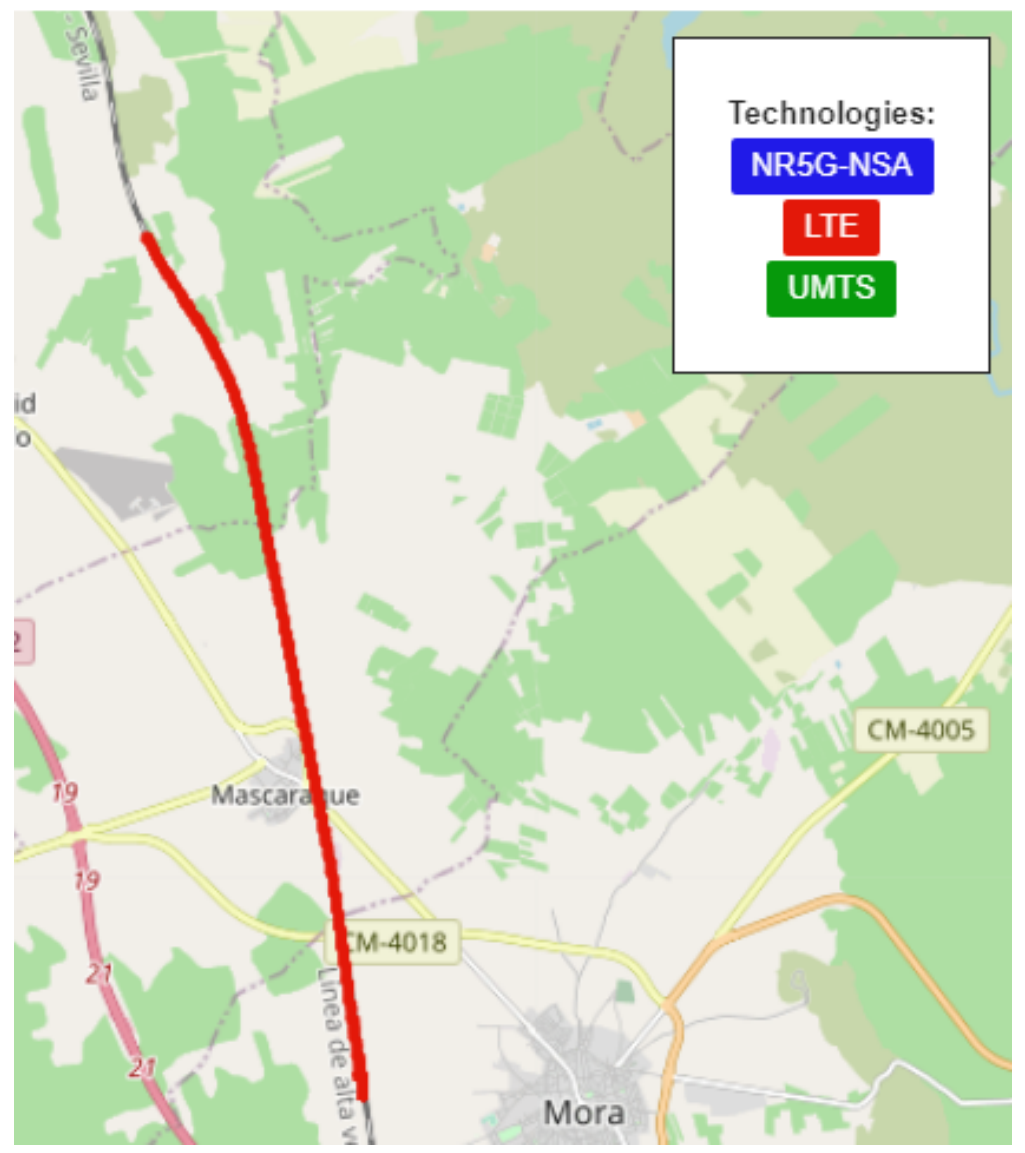

Figure 7. Technology coverage on the track.
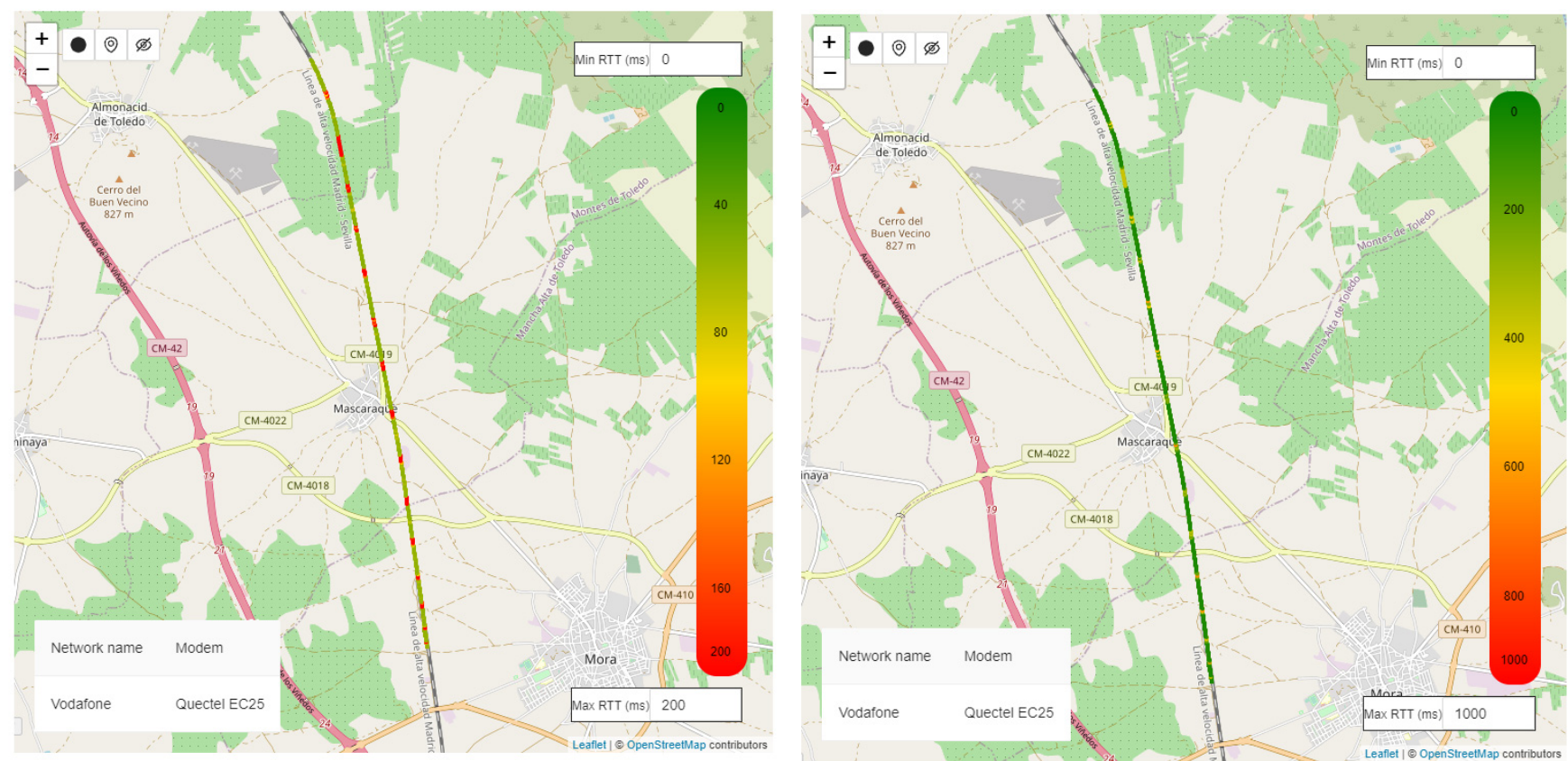

Figure 8. Comparison of the same measurement depending on the KSR of each application (right max RTT 200 ms, left max RTT 7000 ms). 


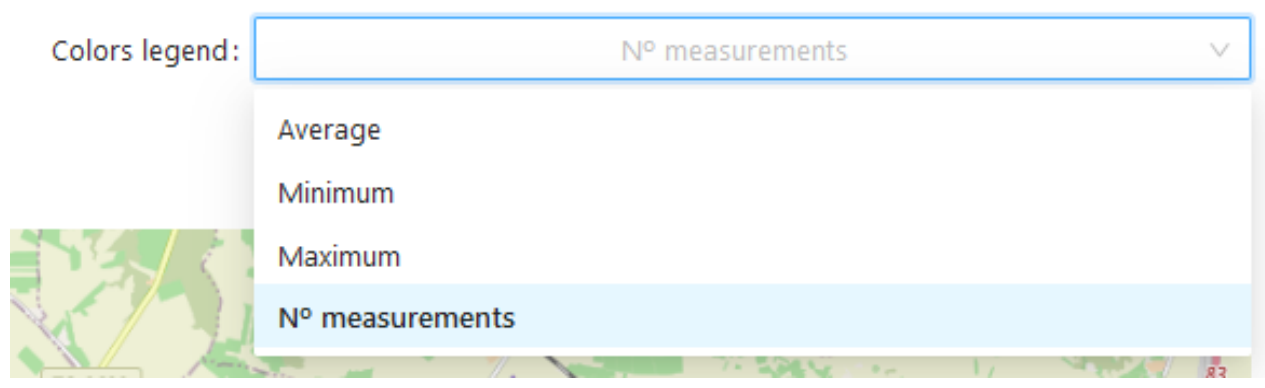

Figure 9. Options to visualize statistics results.

Figure 10 shows the average value from every RTT test performed along the track. In this way, the user can see which average RTT was measured in the selected sized areas. The user knows the average of the RTT, which has been obtained from the performance of the same impairment in different moments in each location of the track. This information can be stated as the summary of the whole communication channel on the track. Based on these results, a decision can be made on which application would fit on the track or which technology would be sufficient to communicate on this track. Apart from the visualization in a map, different charts are available, as shown in Figure 11. This type of chart compares the RTT values with the RF values and the disconnections (CCT Agent had no connection) chart. The RF values in LTE technology are split into different quality indicators: RSSI, SINR, RSRQ, and RSRP.

The charts bring some interesting information for analysis. For example, the bad quality of the signal in the $22 \mathrm{~h} 54 \mathrm{~m}$ time could be the cause of the last disconnection; the SINR is negative and the RSRP is close to $100 \mathrm{dBm}$, which is probably because of being in the cell edge of the cell.

Moreover, if the user has some KSR from an application, it can insert them into the system, and as a result of the application, it will behave correctly or not in the track, as Figure 12 shows. CBTC or ETCS are the applications that are compared in this paper vs. the RTT values along the track.

\section{Statistic Results $\quad$ Statistic Results > Maps}

\begin{tabular}{|c|c|c|c|c|c|c|c|c|}
\hline Ground truth: & & & & Real & & & & $v$ \\
\hline Size accuracy: & $1.2 \mathrm{~km}$ & $v$ & Selection type: & RTT & $v$ & Colors legend: & Average & $v$ \\
\hline
\end{tabular}

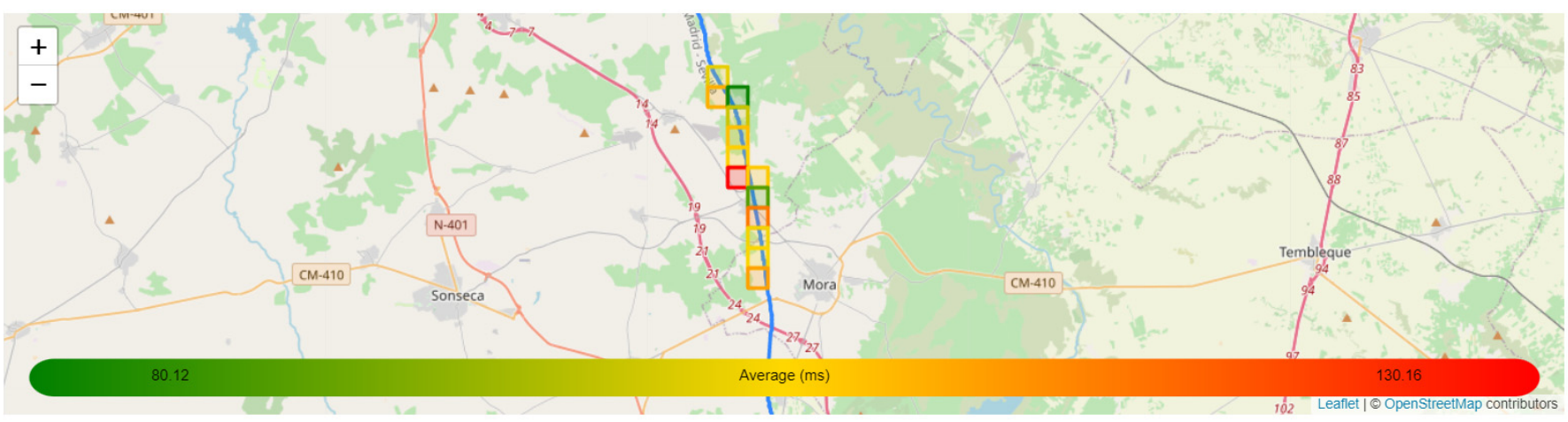

Figure 10. Statistics results from RTT values in the track. 

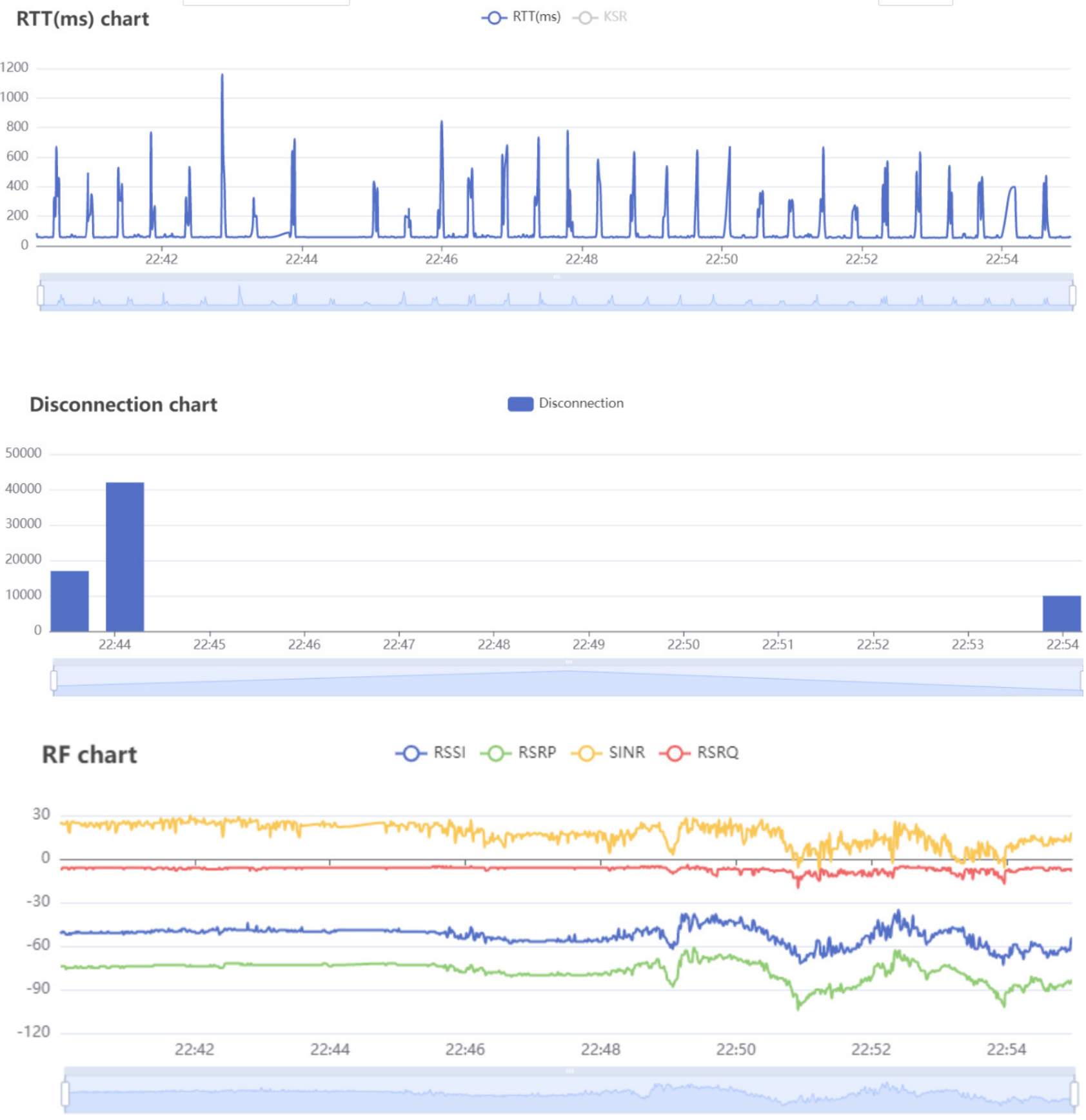

Figure 11. Charts RTT vs. RF values.

Finally, as said throughout the whole paper, the user can test the communication channel to know if the application would behave correctly or not. Regarding Figure 12, the specific applications chosen are ETCS and CBTC. In this case, ETCS would work correctly along this track because the KSR regarding the delay ( $3.5 \mathrm{~s})$ is higher than the measured RTT values. In contrast, the CBTC application would not behave properly due to the peaks of RTT that the track has. 
KSR Type: $\quad$ KSR 1 Max. Latency

RTT(ms) chart
$-\mathrm{O}-\mathrm{RTT}(\mathrm{ms})-\mathrm{O}-\mathrm{KSR}$
Parameter: ETCS
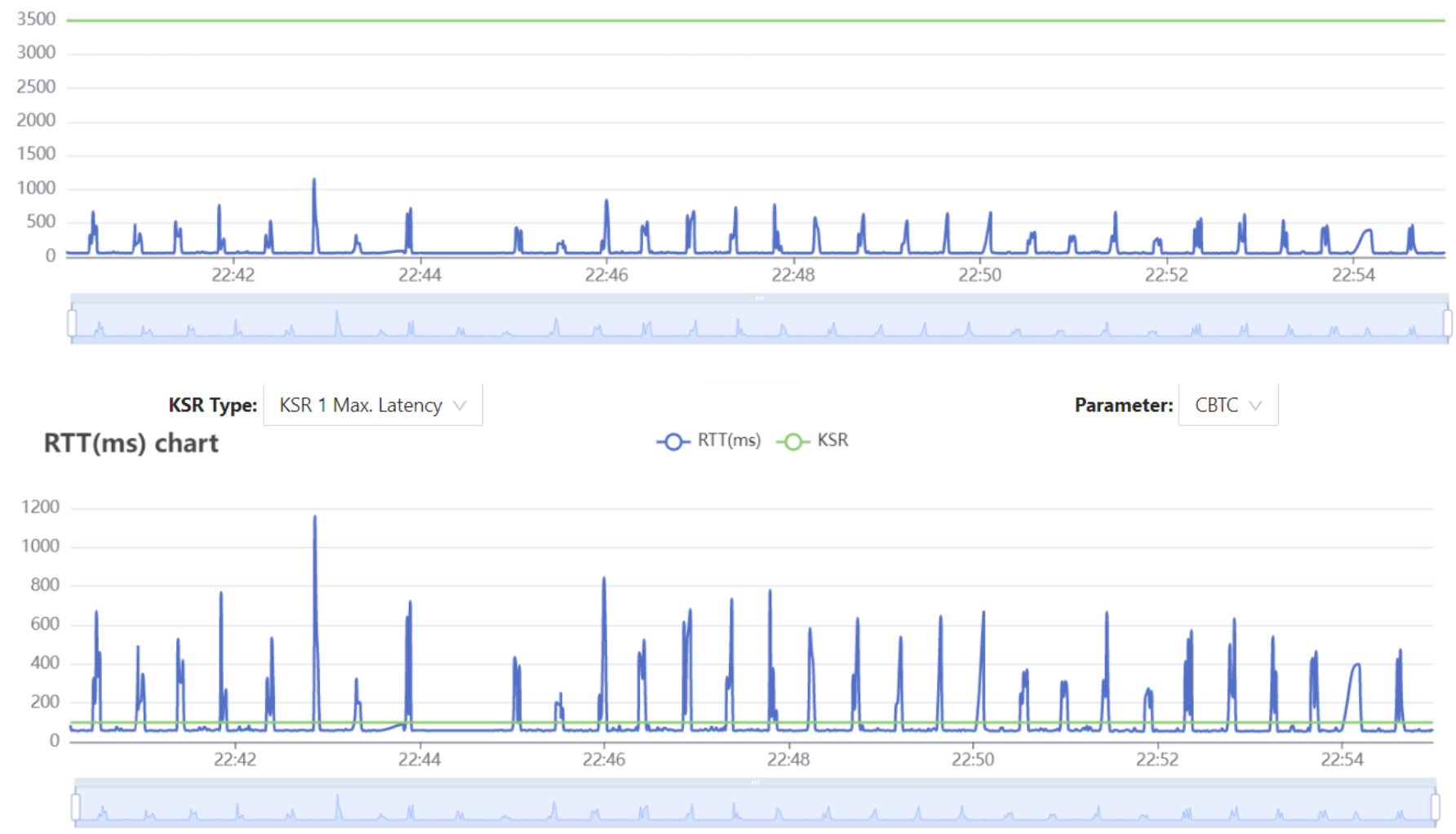

Figure 12. KSR vs. RTT measured value.

\section{Conclusions}

The technology and transportation sectors are in continuous evolution to provide the best experience to the final user. Therefore, new applications and technologies are being implemented and deployed. Among those new developments, it can be stated that CCT has been proven to be a powerful tool that supports the methodology proposed for the communication channel characterization methodology. This methodology can bring the needed information to help understand the behavior of communication technologies in the railway environment and its suitability to migrate the dedicated communication to the public services. This is achieved by analyzing the impairments by means of parameters that normally describe a communication channel such as RTT, bandwidth, or packet loss. Thanks to the special innovative feature of continuous testing, the possibility of measuring these parameters along the whole track is realized. In comparison with the current tools in the market, the CCT is an adaptable tool due to its modularity, allowing the integration of other technologies already deployed but not included or in development. Furthermore, the chance of exchanging the communication antennas in the CCT allows for a possible study of antenna selection for the final user to be carried out due to the feature of plugging different antennas. Additionally, non-communication expert users, such as maintenance operators, are able to use the CCT due to the special easy usage offered by CCT. The proposed methodology brings the possibility of finding out how a given application will work in a real scenario, reducing the learning curve corresponding to the test configuration and visualization of the results.

Obtained results can help to determine why the communication channel varies along the track, e.g., due to the surrounding environment. The effects of the track environment in a selected technology are shown because of the mapping of the measured value with its corresponding location in each moment. Additionally, communication issues can be found 
in any track due to the deployed technology itself or due to the environment that affects the communication channel. With this methodology, these critical areas are identified, which could be a relevant point to be analyzed in the transportation control systems regarding safety issues. Moreover, CCT can also show where an application will have the required QoS, comparing the results with the target KSRs.

In conclusion, this methodology permits both knowing how the communication channel is along the track by means of measuring the performance of the technology (QoS) and determining if a given application would behave correctly at each point of the track in a user-friendly way. Additionally, it provides the characterization of the on-site communication channel for a specific real case having as result inputs for laboratory testing, which will enable shifting the real world to the laboratory environment.

Author Contributions: Conceptualization, N.F.-B., J.G. and J.M.; methodology, N.F.-B., J.G. and J.M.; software, N.F.-B., I.M. and J.A.; validation, N.F.-B. and J.A.; formal analysis, N.F.-B., J.G. and J.A.; investigation, N.F.-B., J.G. and J.M.; writing-original draft preparation, N.F.-B.; writing-review and editing, N.F.-B., J.G., I.M., J.A. and J.M.; visualization, N.F.-B.; supervision, J.M. and J.G.; project administration, J.M. and J.G.; funding acquisition, J.M. All authors have read and agreed to the published version of the manuscript.

Funding: This research has received funding from the European Union's Horizon 2020 research and innovation programme under grant agreement No: 826141: https:/ / projects.shift2rail.org/s2r_ip2_n. aspx?p=X2RAIL-3 (accessed on 19 September 2021).

Institutional Review Board Statement: Not applicable.

Informed Consent Statement: Not applicable.

Data Availability Statement: Not applicable.

Conflicts of Interest: The authors declare no conflict of interest.

\section{References}

1. Moreno, J.; Riera, J.M.; de Haro, L.; Rodriguez, C. A survey on future railway radio communications services: Challenges and opportunities. IEEE Commun. Mag. 2015, 53, 62-68. [CrossRef]

2. He, R.; Ai, B.; Wang, G.; Guan, K.; Zhong, Z.; Molisch, A.F.; Briso-Rodriguez, C.; Oestges, C.P. High-Speed Railway Communications: From GSM-R to LTE-R. IEEE Veh. Technol. Mag. 2016, 11, 49-58. [CrossRef]

3. Dudoyer, S.; Deniau, V.; Heddebaut, M.; Mariscotti, A.; Member, S. Classification of Transient EM Noises Depending on their Effect on the Quality of GSM-R Reception. IEEE Trans. Electromagn. Compat. 2013, 55, 867-874. [CrossRef]

4. Dudoyer, S.; Deniau, V.; Adriano, R.; Ben Slimen, M.N.; Rioult, J.J.; Meyniel, B.; Berbineau, M. Study of the susceptibility of the GSM-R communications face to the electromagnetic interferences of the rail environment. IEEE Trans. Electromagn. Compat. 2012, 54, 667-676. [CrossRef]

5. Chen, R.; Long, W.; Mao, G. Development Trends of Mobile Communication Systems for Railways. IEEE Commun. Surv. Tutor. 2018, 20, 3131-3141. [CrossRef]

6. Calle-sánchez, J.; Molina-garcía, M.; Alonso, J.I.; Fernández-durán, A. Long Term Evolution in High Speed Railway Environments: Feasibility and Challenges. Bell Labs Tech. J. 2013, 18, 237-253. [CrossRef]

7. ITU-T. ITU-T Rec. G.9961: Series G: Transmission Systems and Media Digital Systems and Networks; ITU-T: Geneva, Switzerland, 2018.

8. Prasad, R.S.; Dovrolis, C.; Murray, M.; Claffy, K.C. Bandwidth Estimation: Metrics, Measurement Techniques, and Tools. IEEE Netw. 2003, 17, 27-35. [CrossRef]

9. ITU-T. ITU-T Rec. G.1050: Network Model for Evaluating Multimedia Transmission Performance over Internet Protocol; ITU-T: Geneva, Switzerland, 2016.

10. Gozdecki, J.; Jajszczyk, A.; Stankiewicz, R. Quality of service terminology in IP networks. IEEE Commun. Mag. 2003, 41, 153-159. [CrossRef]

11. ITU-T. ITU-T Rec. K114 Series K: Protection against Interference; ITU-T: Geneva, Switzerland, 2015.

12. 3GPP. Technical Specification: 3GPP TS 36.214 V12.2.0 (2015-03); 3GPP: Sophia Antipolis, France, 2015.

13. Available online: https://www.rohde-schwarz.com/es/producto/romes-pagina-de-inicio-producto_63493-8650.html (accessed on 12 July 2021).

14. Available online: https:/ / www.dingli.com/PilotPioneer.php (accessed on 6 July 2021).

15. Available online: https:// rantcell.com/index.html (accessed on 12 July 2021). 
16. Deniau, V.; Vantrovs, T.; Becuwe, N.; Gransart, C.; Jose, A.N.d.; Boe, A.; Simon, E.P.; Vlamynck, O.; Valenti, F.; Villain, J.; et al. Analysis of the Susceptibility of the LoRa Communication Protocol in the Railway Electromagnetic Environment. In Proceedings of the 2021 XXXIVth General Assembly and Scientific Symposium of the International Union of Radio Science (URSI GASS), Rome, Italy, 28 August-4 September 2021; pp. 1-4.

17. Ghobaei-Arani, M.; Souri, A.; Baker, T.; Hussien, A. ControCity: An Autonomous Approach for Controlling Elasticity Using Buffer Management in Cloud Computing Environment. IEEE Access 2019, 7, 106912-106924. [CrossRef]

18. Shift2Rail Project. X2Rail-1 User E System Requirements (Telecommunications); Shift2 Europe's Rail: Brussels, Belgium, 2016; Volume 2.

19. Fernandez, N.; Arrizabalaga, S.; Añorga, J.; Goya, J.; Adín, I.; Mendizabal, J. Survey of Environmental Effects in Railway Communications. In Lecture Notes in Computer Science; Communication Technologies for Vehicles. Nets4Cars/Nets4Trains/Nets4Aircraft 2018; Springer: Cham, Switzerland, 2018; Volume 10796. [CrossRef]

20. He, D.; Ai, B.; Guan, K.; Yang, Y.; Zhang, L.; Zhong, Z.; Hrovat, A. Significance Analysis for Typical Objects in mmWave Urban Railway Propagation Environment. In Proceedings of the 2017 IEEE Globecom Workshops (GC Wkshps), Singapore, 4-8 December 2017; pp. 1-5.

21. Zhou, T.; Tao, C.; Salous, S.; Liu, L. Measurements and Analysis of Short-Term Fading Behavior in High-Speed Railway Communication Networks. IEEE Trans. Veh. Technol. 2019, 68, 101-112. [CrossRef]

22. He, R.; Zhong, Z.; Ai, B.; Ding, J.; Yang, Y.; Molisch, A.F. Short-Term Fading Behavior in High-Speed Railway Cutting Scenario: Measurements, Analysis, and Statistical Models. IEEE Trans. Antennas Propag. 2013, 61, 2209-2222. [CrossRef]

23. He, R.; Zhong, Z.; Ai, B.; Wang, G.; Ding, J.; Molisch, A.F. Measurements and Analysis of Propagation Channels in High-Speed Railway Viaducts. IEEE Trans. Wirel. Commun. 2013, 12, 794-805. [CrossRef]

24. Zhang, B.; Zhong, Z.; He, R.; Tufvesson, F.; Ai, B. Measurement-Based Multiple-Scattering Model of Small-Scale Fading in High-Speed Railway Cutting Scenarios. IEEE Antennas Wirel. Propag. Lett. 2017, 16, 1427-1430. [CrossRef]

25. He, R.; Zhong, Z.; Ai, B.; Ding, J. Propagation measurements and analysis for high-speed railway cutting scenario. In Proceedings of the 2012 IEEE Global Communications Conference (GLOBECOM), Anaheim, CA, USA, 3-7 December 2012; Volume 47, pp. 17-18.

26. Guan, K.; Zhong, Z.; Ai, B.; Member, S.; Kürner, T.; Member, S. Empirical Models for Extra Propagation Loss of Train Stations on High-Speed Railway. IEEE Trans. Antennas Propag. 2014, 62, 1395-1408. [CrossRef]

27. Ke, W.; Suoping, L.I.; Ying, L.I.N.; Zufang, D.O.U.; Wei, L.I. Performance Analysis of High-speed Railway Handover Scheme with Different Network Architecture. In Proceedings of the 2019 IEEE 8th Joint International Information Technology and Artificial Intelligence Conference (ITAIC), Chongqing, China, 24-26 May 2019; pp. 1894-1898.

28. Wang, J.; Zhang, D. The Influence of Pantograph Arcing Radiation Disturbance on LTE-R. In Proceedings of the 2019 International Conference on Electromagnetics in Advanced Applications (ICEAA), Granada, Spain, 9-13 September 2019; pp. $0583-0586$.

29. Stienne, O.; Deniau, V.; Simon, E.P.; Kousri, M.R. Susceptibility of 4 G communications in railway EM environment. In Proceedings of the 2018 2nd URSI Atlantic Radio Science Meeting (AT-RASC), Gran Canaria, Spain, 28 May-1 June 2018; pp. $2018-2021$.

30. Sniady, A.; Soler, J. LTE for Railways: Impact on Performance of ETCS Railway Signaling. IEEE Veh. Technol. Mag. 2014, 9, 69-77.

31. Bist, A.; Singhal, C. Efficient Immersive Surveillance of Inaccessible Regions using UAV Network. In Proceedings of the IEEE INFOCOM 2021-IEEE Conference on Computer Communications Workshops (INFOCOM WKSHPS), Vancouver, BC, Canada, 10-13 May 2021; pp. 1-6. 